EPSC Abstracts

Vol. 14, EPSC2020-270, 2020

https://doi.org/10.5194/epsc2020-270

Europlanet Science Congress 2020

(C) Author(s) 2020. This work is distributed under

the Creative Commons Attribution 4.0 License.

\title{
Flying through a dawn storm: a multi-instrument study of the traversal of a dawn storm by Juno on February 7th 2018
}

\author{
Bertrand Bonfond ${ }^{1}$, Ruilong Guo ${ }^{1}$, Zhonghua $\mathrm{Yao}^{2}$, Grodent Denis ${ }^{1}$, Jean-Claude Gérard ${ }^{1}$, \\ Gladstone Randy ${ }^{3}$, Hue Vincent ${ }^{3}$, Greathouse Thomas ${ }^{3}$, Maarten Versteeg ${ }^{3}$, Joshua Kammer ${ }^{3}$, \\ Frederic Allegrini ${ }^{3}$, Robert Ebert ${ }^{3}$, William Kurth ${ }^{4}$, Philippe Louarn ${ }^{5}$, Daniel Gershman ${ }^{6}$, John \\ Connerney $^{6}$, Stavros Kotsiaros ${ }^{7}$, and Barry Mauk ${ }^{8}$ \\ ${ }^{1}$ Laboratory for Planetary and Atmospheric Physics, STAR Institute, University of Liège, Liège, Belgium (b.bonfond@uliege.be) \\ ${ }^{2}$ Key laboratory of Earth and Planetary Physics, Institute of Geology and Geophysics, Chinese Academy of Sciences, Beijing, \\ China \\ ${ }^{3}$ Southwest Research Institute, San Antonio, TX, USA \\ ${ }^{4}$ Department of Physics and Astronomy, University of Iowa, Iowa City, IA, USA. \\ ${ }^{5}$ IRAP, Toulouse, France \\ ${ }^{6}$ NASA Goddard Spaceflight Center, Greenbelt, Maryland, USA \\ ${ }^{7}$ DTU Space, Denmark \\ ${ }^{8}$ Applied Physics Laboratory, Johns Hopkins University, Laurel, MD, USA.
}

On February 7th 2018, during Juno's 11th perijove observation sequence, Juno's ultraviolet spectrograph (Juno-UVS) unveiled the development of a dawn storm in Jupiter's aurorae. These auroral events consist of spectacular brightenings of the midnight to dawn sector of the main emissions at Jupiter. At the end of the sequence, Juno crossed the magnetic field lines connected to this dawn storm, unraveling some of the processes giving rise to these spectacular events.

All in situ instruments detected a sharp transition as the spacecraft entered the dawn storm at an altitude of approximately 5RJ in the southern hemisphere. The particle fluxes detected by the JADE and JEDI instruments, including electrons and ions, increased dramatically. A strong flux of penetrating radiation was also detected by the UVS instrument. The Alfvén waves spectrograms derived from the MAG instrument also show a clear transition between a quiet and an extremely active regime as the spacecraft entered the dawn storm. Furthermore, the orientation of the magnetic field showed a very strong perturbation, associated with intense currents. And, finally, intense bKOM emissions were also observed during this time interval. Combined with the remote sensing observations of the aurora, these datasets strongly suggest that Juno witnessed a strong magnetospheric reconfiguration that started in the magneto-tail and then evolved toward dawn as the planet rotated. 